\title{
Engineering transcription factors to improve tolerance against alkane biofuels in Saccharomyces cerevisiae
}

Hua Ling ${ }^{1,2}$, Nina Kurniasih Pratomo Juwono ${ }^{1,2}$, Wei Suong Teo ${ }^{1,2}$, Ruirui Liu ${ }^{1,2}$, Susanna Su Jan Leong ${ }^{1,2,3}$ and Matthew Wook Chang ${ }^{1,2^{*}}$

\begin{abstract}
Background: Biologically produced alkanes can be used as 'drop in' to existing transportation infrastructure as alkanes are important components of gasoline and jet fuels. Despite the reported microbial production of alkanes, the toxicity of alkanes to microbial hosts could pose a bottleneck for high productivity. In this study, we aimed to improve the tolerance of Saccharomyces cerevisiae, a model eukaryotic host of industrial significance, to alkane biofuels.

Results: To increase alkane tolerance in S. cerevisiae, we sought to exploit the pleiotropic drug resistance (Pdr) transcription factors Pdr1 $p$ and Pdr3p, which are master regulators of genes with pleiotropic drug resistance elements (PDREs)-containing upstream sequences. Wild-type and site-mutated Pdr1 $\mathrm{p}$ and Pdr3p were expressed in S. cerevisiae BY4741 pdr1 $\triangle p d r 3 \triangle$ (BYL13). The point mutations of PDR1 (F815S) and PDR3 (Y276H) in BYL13 resulted in the highest tolerance to C10 alkane, and the expression of wild-type PDR3 in BYL13 led to the highest tolerance to C11 alkane. To identify and verify the correlation between the Pdr transcription factors and tolerance improvement, we analyzed the expression patterns of genes regulated by the Pdr transcription factors in the most tolerant strains against C10 and C11 alkanes. Quantitative PCR results showed that the Pdr transcription factors differentially regulated genes associated with multi-drug resistance, stress responses, and membrane modifications, suggesting different extents of intracellular alkane levels, reactive oxygen species (ROS) production and membrane integrity. We further showed that (i) the expression of $\mathrm{Pdr}_{\mathrm{mt}}+\mathrm{Pdr} 3_{\mathrm{mt}}$ reduced intracellular $\mathrm{C} 10$ alkane by $67 \%$ and ROS by $53 \%$, and significantly alleviated membrane damage; and (ii) the expression of the Pdr $3_{w t}$ reduced intracellular C11 alkane by $72 \%$ and ROS by $21 \%$. Alkane transport assays also revealed that the reduction of alkane accumulation was due to higher export (C10 and C11 alkanes) and lower import (C11 alkane).
\end{abstract}

Conclusions: We improved yeast's tolerance to alkane biofuels by modulating the expression of the wild-type and site-mutated Pdr1 $p$ and Pdr3p, and extensively identified the correlation between Pdr transcription factors and tolerance improvement by analyzing gene patterns, alkane transport, ROS, and membrane integrity. These findings provide valuable insights into manipulating transcription factors in yeast for improved alkane tolerance and productivity.

Keywords: Pleiotropic drug resistance, Transcription factors, Site mutagenesis, Alkanes, Biofuels, Tolerance, Saccharomyces cerevisiae

\footnotetext{
*Correspondence: bchcmw@nus.edu.sg

${ }^{1}$ Department of Biochemistry, Yong Loo Lin School of Medicine, National

University of Singapore, 8 Medical Drive, 117597 Singapore, Singapore

Full list of author information is available at the end of the article
} 


\section{Background}

Biologically synthesized alkanes can be used as drop in' to existing transportation infrastructure as alkanes are important components of gasoline and jet fuels [1]. Even though alkanes have been successfully produced in microbes [2-8], the yields and titers should be a key consideration for industrial-scale production, and the toxicity of alkanes to microbial hosts could eventually be a bottleneck for high productivity $[9,10]$.

Our previous transcriptome analyses suggested that alkanes induce a range of cellular mechanisms such as efflux pumps, membrane modification, radical detoxification, and energy supply in yeast [9]. Indeed, the mechanisms underlying cell responses to toxic molecules can provide useful strategies to improve cell tolerance and viability. Such strategies include engineering efflux pumps [9-12] and transcription factors [13-17], and modifying cellular membrane [18]. Transcription factors regulate multiple and simultaneous perturbations of the transcriptome towards a global phenotype of tolerance [19]. By knockout or overexpression of transcription factors involved in genetic regulatory networks of isooctane response in Escherichia coli, Kang et al. [20] improved E. coli's tolerance to isooctane. In addition, Matsui et al. discovered a modified transcription factor endowing Saccharomyces cerevisiae with organic-solvent tolerance [21].

Towards the development of alkane-tolerant S. cerevisiae, a well-studied model eukaryote with wide industrial applications, we sought to exploit its transcription factors Pdr1p and Pdr3p, which are master regulators of genes with pleiotropic drug resistance elements (PDREs)-containing upstream sequences [22]. Currently, a thorough investigation of the roles of Pdr1p and Pdr3p in cellular tolerance to alkanes is lacking. In this study, we demonstrated a significant improvement in yeast's tolerance to $\mathrm{n}$-decane (C10) and n-undecane (C11) by modulating the expression of wild-type and site-mutated Pdr1p and Pdr3p. The correlation between Pdr transcription factors and tolerance improvement was confirmed by analyzing gene patterns, alkane transport, reactive oxygen species (ROS) levels, and membrane integrity.

\section{Results and discussion}

\section{Site-mutation of PDR 1 and PDR3}

Transcription factor engineering is widely used to improve microbial strain tolerance against toxic molecules $[12,14]$. In $S$. cerevisiae, transcription factors Pdr1p and Pdr3p have a DNA-binding domain, an inhibitory domain, and a transcription activation domain. The inhibitory domain in a locked conformation interacts with the transcription activation domain [23, 24], which is associated with Pdr-DNA or Pdr-Pdr interactions and pleiotropic drug resistance. Amino acid substitutions in the inhibitory domains could alter the actions of the transcription activation domain, leading to changes in Pdr1 and Pdr3 activity and the pleiotropic drug resistance. Recently, a series of site mutations in the inhibitory domains have been shown to improve pleiotropic drug resistance, and three site mutations (F815S and R821S in Pdr1p, and Y276H in Pdr3p) are most effective to improve the tolerance against various toxic molecules [21, 23-25]. Currently, a thorough investigation of the roles of these mutations in cellular tolerance to alkanes is lacking. In this study, we chose F815S and R821S in Pdr1p and $\mathrm{Y} 276 \mathrm{H}$ in Pdr3p for improving the tolerance to alkane biofuels in S. cerevisiae. Figure 1 shows the chosen mutation sites and cloning of the wild-type and sitemutated PDR 1 and PDR3 into pESC-Ura. We induced the expression of the wild-type and site-mutated PDR1 and PDR3 in a double gene-deletion mutant S. cerevisiae BYL13 ( $p d r 1 \Delta p d r 3 \Delta)$.

\section{Conditions for protein induction and alkane exposure}

To test yeast cell tolerance towards alkanes, we determined suitable conditions for protein induction and alkane exposure. Additional file 1: Figure S1A shows that the growth of BYL13 expressing the site-mutated Pdr transcription factor genes was inhibited, suggesting that lower induction of the Pdr transcription factors might lead to lower growth inhibition. To determine suitable induction conditions, we added various amounts of galactose $(0.5 \mathrm{~g} / \mathrm{l}, 5 \mathrm{~g} / \mathrm{l}$, and $20 \mathrm{~g} / \mathrm{l})$ and compared the resulting cell densities. Additional file 1: Figure S1B shows that the growth inhibition was lower (one-tailed Student $t$ test, $\mathrm{p}<0.05)$ at $24 \mathrm{~h}$ when the expression was induced by a lower concentration of galactose $(0.5 \mathrm{~g} / \mathrm{l})$. Hence, we chose to use $0.5 \mathrm{~g} / \mathrm{l}$ of galactose for the induction of transcription factor expression in further experiments.

Medium chain alkanes (C8-C12) are important components of transportation fuels such as gasoline and jet fuels. As C12 alkane exhibits insignificant toxicity to $S$. cerevisiae [10], we aimed to improve yeast tolerance towards $\mathrm{C} 8, \mathrm{C} 9, \mathrm{C} 10$, and $\mathrm{C} 11$ alkanes. Additional file 1 : Figure $\mathrm{S} 2$ shows that $5 \%$ of $\mathrm{C} 8, \mathrm{C} 9$, or $\mathrm{C} 11$ alkanes, and $1 \%$ of C10 alkane inhibited the growth of BYL13 carrying empty pESC-Ura plasmid. These alkane concentrations, together with $0.5 \mathrm{~g} / \mathrm{l}$ of galactose, were used for determining engineered yeast tolerance towards alkanes.

\section{Tolerance of BYL13 expressing Pdr transcription factors towards alkanes}

We then investigated the tolerance of BYL13 expressing Pdr transcription factors against C8, C9, C10, and C11 alkanes. Figure 2a and Additional file 1: Figure S3 show that in the presence of (i) $1 \%$ C10 alkane, BYL13 

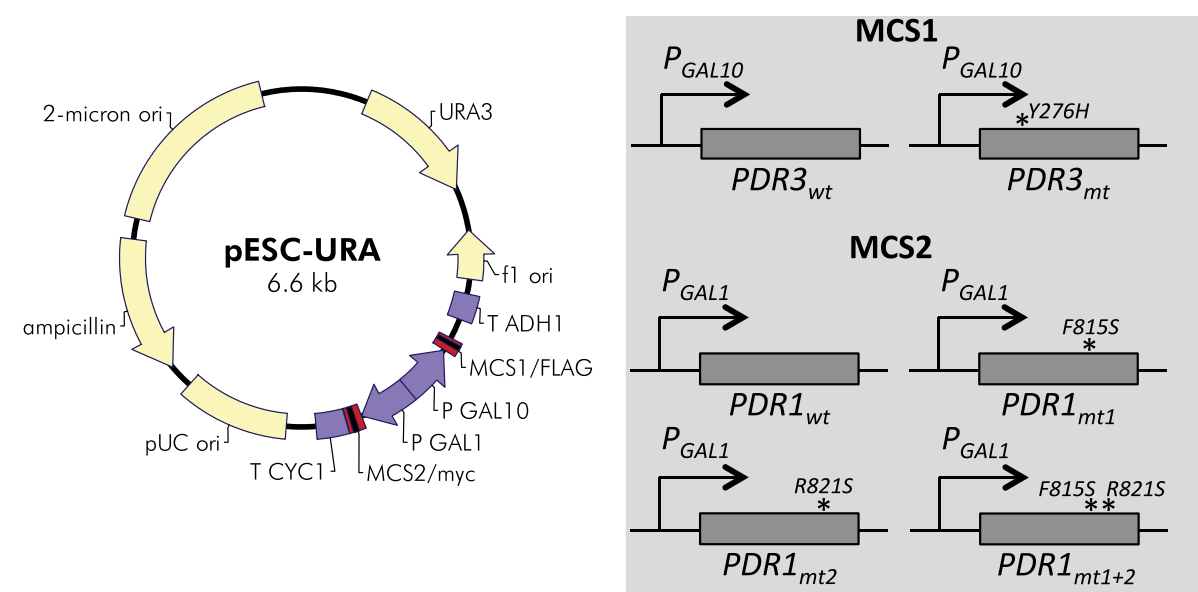

Fig. 1 Site mutagenesis of PDR1 and PDR3, and plasmid construction. Plasmid pESC-Ura (http://www.chem-agilent.com) was used as a vector to express the transcription factors. Wild-type and mutant alleles of PDR1 and PDR3 were cloned into MCS2 and MCS1, respectively. Asterisks mutation sites
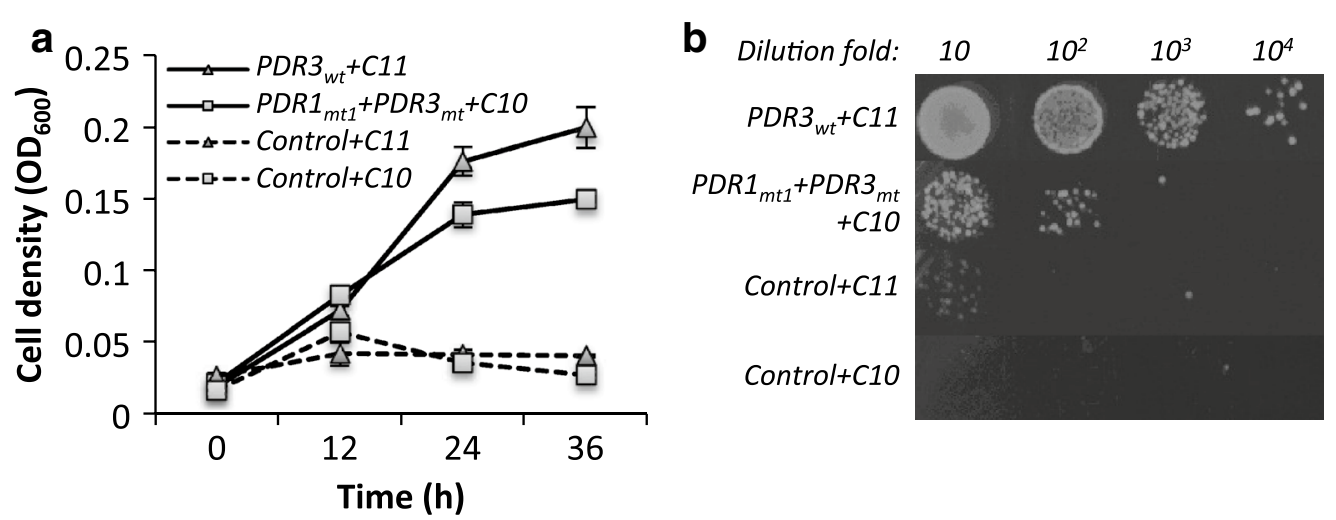

Fig. 2 Tolerance of BYL13 $+\mathrm{Pdr}\left(\mathrm{Pdr} 1_{\mathrm{mt1}}+\mathrm{Pdr} 3_{\mathrm{mt}}\right.$ and $\left.\mathrm{Pdr} 3_{\mathrm{wt}}\right)$ against $\mathrm{C} 10$ and $\mathrm{C} 11$ alkanes. a Cell density $\left(\mathrm{OD}_{600}\right)$ of BYL13 expressing $P d r 1_{m t 1}+P d r 3_{m t}$ against $1 \% C 10\left(P D R 1_{m t 1}+P D R 3_{m t}+C 10\right)$, and BYL13 expressing Pdr3 $3_{w t}$ against $5 \%$ C11 (PDR3 $\left.{ }_{w t}+C 11\right)$ was measured every $12 \mathrm{~h}$. Error bars SD from three biological replicates. b Ten microliters of serially diluted cells ( $24 \mathrm{~h}$ ) were spotted onto YPD agar plates for cell viability assays. Control, BYL13 with pESC-Ura

expressing the site-mutated Pdr transcription factors (particularly $\mathrm{Pdr} 1_{\mathrm{mt1}}+\mathrm{Pdr} 3_{\mathrm{mt}}$ ) had significantly higher cell densities than the control cells (with pESC-Ura); and (ii) $5 \%$ C11 alkane, BYL13 expressing the wild-type Pdr transcription factors (particularly Pdr3 $3_{\mathrm{wt}}$ ) had significantly higher cell densities, whilst BYL13 expressing the site-mutated Pdr transcription factors had modestly higher cell densities than the controls. The enhanced cell densities correspond with increased cell viability (Fig. 2b). However, there was no improvement in tolerance in BYL13 expressing the wild-type Pdr transcription factors against C10 alkane, or in BYL13 expressing any Pdr transcription factors against $\mathrm{C} 8$ or $\mathrm{C} 9$ alkanes. Expression of the representative transcription factors $\left(\operatorname{Pdr} 1_{\mathrm{mt} 1}+\mathrm{Pdr} 3_{\mathrm{mt}}\right.$, and $\left.\mathrm{Pdr} 3_{\mathrm{wt}}\right)$ was confirmed by
Western blots (Additional file 1: Figure S4). Furthermore, we performed quantitative PCR (qPCR) to understand the roles of $P D R$ expression levels and site mutations in the tolerance improvement. Additional file 2: Tables S1 and $\mathrm{S} 2$ show that the $\mathrm{C} 10$ tolerance was attributed to the site mutations (Pdr1 F815S, and Pdr3 Y276H) regardless of the $P D R 1_{m t 1}$ and $P D R 3_{m t}$ expression levels. The results of the growth assays and expression analyses indicate that the expression of Pdr transcription factors improved yeast tolerance to $\mathrm{C} 10$ and $\mathrm{C} 11$ alkanes.

Gene patterns in BYL13 expressing Pdr $1_{m t 1}+\mathrm{Pdr}_{\mathrm{mt}}$ and $\mathrm{Pdr}_{\mathrm{wt}}$ in the presence of $\mathrm{C} 10$ and $\mathrm{C} 11$ alkanes

The improved alkane tolerance of yeast, conferred by the expression of $\mathrm{Pdr} 1_{\mathrm{mt} 1}+\operatorname{Pdr} 3_{\mathrm{mt}}$ or $\mathrm{Pdr} 3_{\mathrm{wt}}$, might be 
contributed to by perturbations made to the expression levels of the genes regulated by the Pdr transcription factors, such as $A B C$ efflux pump genes, stress responsive genes, and genes involved in membrane modifications [22]. To examine this possibility, we studied the expression patterns of those target genes in the presence of $\mathrm{C} 10$ and $\mathrm{C} 11$ alkanes by qPCR.

First, to choose a suitable reference gene, we evaluated expression stability of five reference genes (ACT1, ALG9, TAF10, UBC6, and TFC1) by comparing their $\mathrm{M}$ values under the above conditions. Here, a lower $M$ value stands for higher stability of gene expression [26, 27]. Additional file 2: Table S1 shows that UBC6 gene had the lowest $M$ value out of the five reference gene candidates under the conditions of Pdr expression and alkane exposure. Hence, $U B C 6$ was chosen as the reference gene for qPCR analyses.

Second, we compared expression levels of the target genes in BYL13 expressing Pdr $1_{\mathrm{mt} 1}+\mathrm{Pdr} 3_{\mathrm{mt}}$ (in the presence of $\mathrm{C} 10$ alkane) or $\mathrm{Pdr}_{\mathrm{wt}}$ (in the presence of $\mathrm{C} 11$ alkane) to those in BYL13 under exposure to $\mathrm{C} 10$ or $\mathrm{C} 11$ alkane. Figure 3 shows that, in BYL13 expressing either Pdr $1_{\mathrm{mt1}}+\operatorname{Pdr} 3_{\mathrm{mt}}$ (C10) or Pdr3 ${ }_{\mathrm{wt}}$ (C11), (i) ABC efflux pump genes (i.e., YOR1, SNQ2, PDR5, and PDR15) were up-regulated by 4.2 to 46.6 -fold (C10) and 1.6 to 17.1-fold (C11); (ii) cytosolic catalase gene CTT1 was up-regulated by 2.1-fold (C10) and 2.6-fold (C11); and (iii) lysophosphatidic acid acyltransferase gene ICT1 was up-regulated by 5.8 -fold (C10) and 3.4-fold (C11). Considering the roles of the efflux pump genes in multi-drug resistance (MDR) [28], CTT1 in ROS detoxification [29], and ICT1 in membrane modifications [18], we hypothesized

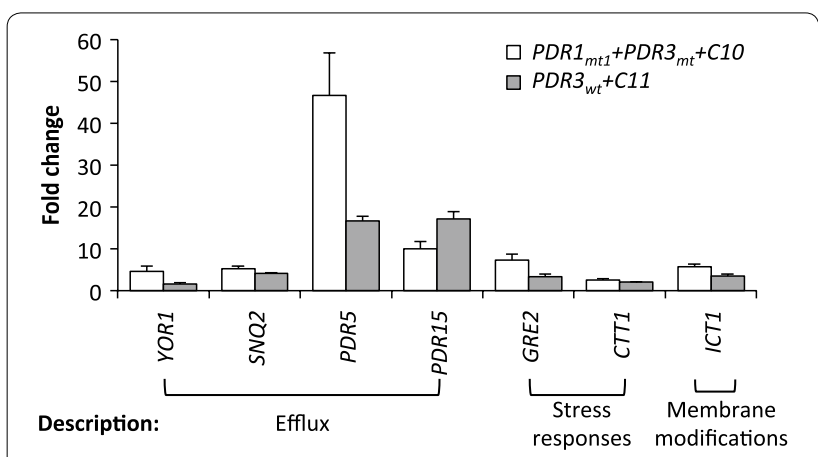

Fig. 3 Relative gene expression levels under the regulation of Pdr transcription factors in the presence of $\mathrm{C} 10$ and $\mathrm{C} 11$ alkanes. In the induction medium containing $0.5 \mathrm{~g} / \mathrm{l}$ galactose, BYL13 + Pdr1 $1_{\mathrm{mt} 1}+$ Pdr $3_{\mathrm{mt}}$ cells were exposed to $1 \% \mathrm{C} 10$ alkane $\left(P D R 1_{m t 1}+P D R 3_{m t}+C 10\right)$, and BYL13 + Pdr $3_{w t}$ cells were exposed to $5 \% \mathrm{C} 11$ alkane (PDR3 $\left.{ }_{w t}+\mathrm{C} 11\right)$. The expression levels of genes at $24 \mathrm{~h}$ were normalized to those in controls (BYL13 + pESC-Ura + C10, $\mathrm{BYL13}+\mathrm{pESC}-$ Ura $+\mathrm{C} 11$ ) and the reference gene UBC6. Error bars SD from three biological replicates that intracellular alkane amount, ROS levels, the efficiency of alkane transport, and membrane damage might be involved in the increased alkane tolerance of BYL13 expressing $\operatorname{Pdr} 1_{\mathrm{mt} 1}+\operatorname{Pdr} 3_{\mathrm{mt}}$ or $\operatorname{Pdr} 3_{\mathrm{wt}}$. This hypothesis was investigated, as explained in the following sections.

\section{Alkane levels in BYL13 expressing Pdr $1_{m t 1}+P d r 3_{m t}$ and Pdr3 ${ }_{\text {wt }}$}

To investigate the effect of the expression of $\operatorname{Pdr} 1_{\mathrm{mt} 1}+\operatorname{Pdr} 3_{\mathrm{mt}}$ or Pdr3 $3_{\mathrm{wt}}$ on intracellular alkane levels, we quantified intracellular $\mathrm{C} 10$ and $\mathrm{C} 11$ alkanes by gas chromatography (GC) analyses. Figure 4a shows that (i) upon exposure to $\mathrm{C} 10$ alkane, the intracellular $\mathrm{C} 10$ alkane amount was reduced by $67 \%$ in BYL13 expressing $\operatorname{Pdr} 1_{\mathrm{mt} 1}+\operatorname{Pdr} 3_{\mathrm{mt}}$; and (ii) upon exposure to C11 alkane, the intracellular C11 alkane amount was reduced by $72 \%$ in BYL13 expressing Pdr3 ${ }_{\mathrm{wt}}$.

Given that $\mathrm{C} 10$ and $\mathrm{C} 11$ alkanes are not metabolized by S. cerevisiae, we hypothesized that the lower intracellular levels of alkanes in BYL13 expressing Pdr transcription factors could be due to alkanes efflux, reduced import, or both. First, to investigate the involvement of alkane efflux in reducing alkane accumulation, we exposed BYL13 expressing $\mathrm{Pdr} 1_{\mathrm{mt1}}+\mathrm{Pdr} 3_{\mathrm{mt}}$ to $\mathrm{C} 10$ alkane and BYL13 expressing Pdr3 $3_{w t}$ to $\mathrm{C} 11$ alkane. Following this, the $\mathrm{ABC}$ efflux pumps were deactivated, and the intracellular alkane amount with and without active $A B C$ efflux pumps was compared. Here, $\mathrm{NaN}_{3}$ functions as a metabolic inhibitor that interferes with $\mathrm{ABC}$ transporters. The $A B C$ transporters in the cells without $\mathrm{NaN}_{3}$ treatment are still active in alkane efflux. Under the condition of no $\mathrm{NaN}_{3}$ treatment, reduction of the intracellular alkane amounts is attributed to alkane efflux associated with the $A B C$ efflux pumps, vise versa. Figure $4 \mathrm{~b}$ shows that in comparison to the alkane levels in BYL13, C10 and C11 alkane levels in BYL13 with active ABC efflux pumps were reduced by 19.9 and $34.5 \%$, respectively. This result suggests significant reduction in intracellular $\mathrm{C} 10$ and $\mathrm{C} 11$ alkanes was contributed by ABC efflux pumps-associated alkanes export. Second, to verify whether lower import of alkanes contributed to reducing intracellular alkane levels, we added $\mathrm{NaN}_{3}$ to deactivate $\mathrm{ABC}$ efflux pumps, and then exposed the cells to C10 and $\mathrm{C} 11$ alkanes, and quantified intracellular alkanes. Upon the deactivation of the $\mathrm{ABC}$ transporters by $\mathrm{NaN}_{3}$, there is no $A B C$ efflux pumps-associated alkane efflux during alkane import. Under this condition, lower intracellular alkane amounts are attributed to lower alkane import, vise versa. Figure 4c shows that, in comparison to the control without Pdr transcription factors, (i) C10 alkane amount was comparable in BYL13 expressing $\mathrm{Pdr} 1_{\mathrm{mt} 1}+\mathrm{Pdr} 3_{\mathrm{mt}}$, suggesting no difference in $\mathrm{C} 10$ alkane import despite the up-regulation of ICT1 in BYL13 


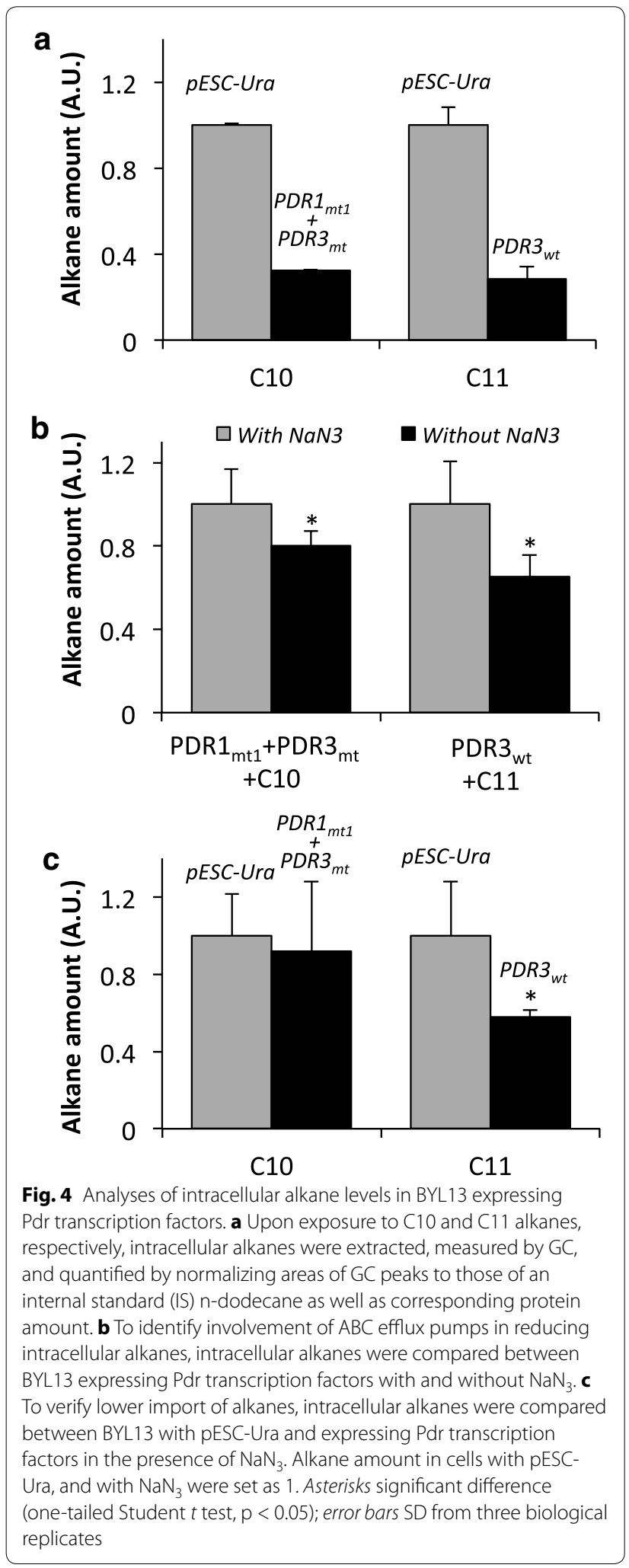

expressing $\mathrm{Pdr} 1_{\mathrm{mt} 1}+\mathrm{Pdr} 3_{\mathrm{mt}}$; and (ii) $\mathrm{C} 11$ alkane amount in BYL13 expressing Pdr $3_{\text {wt }}$ was reduced by $42.2 \%$, suggesting lower import of $\mathrm{C} 11$, which is consistent with the up-regulation of ICT1. Hence, these results suggest that alkane efflux might contribute to $\mathrm{C} 10$ reduction, and both alkane efflux and low import to $\mathrm{C} 11$ reduction.

ROS levels in BYL13 expressing Pdr1 $1_{m+1}+\mathrm{Pdr}_{\mathrm{mt}}$ and $\mathrm{Pdr} 3_{\text {wt }}$ with $\mathrm{C} 10$ and $\mathrm{C} 11$ alkanes

ROS levels were quantified to investigate the effect of Pdr transcription factor expression on ROS production in the presence of alkanes. Figure $5 \mathrm{a}, \mathrm{b}$ shows that C10 alkane enhanced ROS levels by more than fourfold whereas $\mathrm{C} 11$ alkane increased ROS levels by 1.5 -fold. Further, in comparison to BYL13 carrying pESC-Ura, intracellular ROS was reduced by $53 \%$ in BYL13 expressing $\operatorname{Pdr} 1_{\mathrm{mt} 1}+\operatorname{Pdr} 3_{\mathrm{mt}}$ in the presence of $\mathrm{C} 10$ alkane, and reduced by $21 \%$ in BYL13 expressing $\operatorname{Pdr} 3_{\mathrm{wt}}$ in the presence of $\mathrm{C} 11$ alkane. The reduction of ROS in BYL13 expressing the Pdr transcription factors was further supported by our microscopy results. Figure $5 \mathrm{c}$ shows that, upon exposure to C10 alkane, over $90 \%$ of the cells with pESC-Ura fluoresced in green, and only about $30 \%$ of the cells with $\mathrm{Pdr} 1_{\mathrm{mt1}}+\mathrm{Pdr} 3_{\mathrm{mt}}$ fluoresced in green. On the other hand, upon exposure to $\mathrm{C} 11$ alkane, $15 \%$ of the cells with pESC-Ura fluoresced in green, and no cells with Pdr $3_{w t}$ fluoresced in green. Here, more green cells and higher fluorescence intensities represent more ROS. The results of ROS quantification and microscopy suggest significant reduction of ROS in BYL13 expressing the Pdr transcription factors in the presence of $\mathrm{C} 10$ and C11 alkanes.

Membrane integrity of BYL13 expressing Pdr $1_{m t 1}+P d r 3_{m t}$ and $\mathrm{Pdr}_{\mathrm{wt}}$ in the presence of $\mathrm{C} 10$ and $\mathrm{C} 11$ alkanes

The qPCR results in Fig. 3 show that ICT1, associated with membrane modifications, was up-regulated in BYL13 expressing Pdr transcription factors upon exposure to $\mathrm{C} 10$ and $\mathrm{C} 11$ alkanes, suggesting that membrane integrity might be affected upon exposure to alkanes [30]. Here, we aimed to study the membrane integrity of BYL13 expressing Pdr transcription factors in the presence of alkanes.

To this end, we exposed the cells to C10 and C11 alkanes and stained the exposed cells with fluorescence nucleic acid stains PI and SYTO 9. Subsequently, we measured fluorescence signals and observed the cells under microscope. Figure $6 \mathrm{a}$ shows that relative fluorescence unit (RFU) ratios of PI to SYTO 9 were enhanced by 16.7 -fold in BYL13 with pESC-Ura, and enhanced by 6.4-fold in BYL13 expressing Pdr $1_{\mathrm{mt} 1}+\operatorname{Pdr} 3_{\mathrm{mt}}$, upon exposure to $\mathrm{C} 10$ alkane as compared with those without alkane exposure. Moreover, in the presence of $\mathrm{C} 10$ alkane, the RFU ratio in BYL13 expressing Pdr $1_{\mathrm{mt} 1}+\mathrm{Pdr} 3_{\mathrm{mt}}$ was about $62 \%$ lower than that with pESC-Ura, likely due to Ict1p-mediated membrane modifications in the presence 

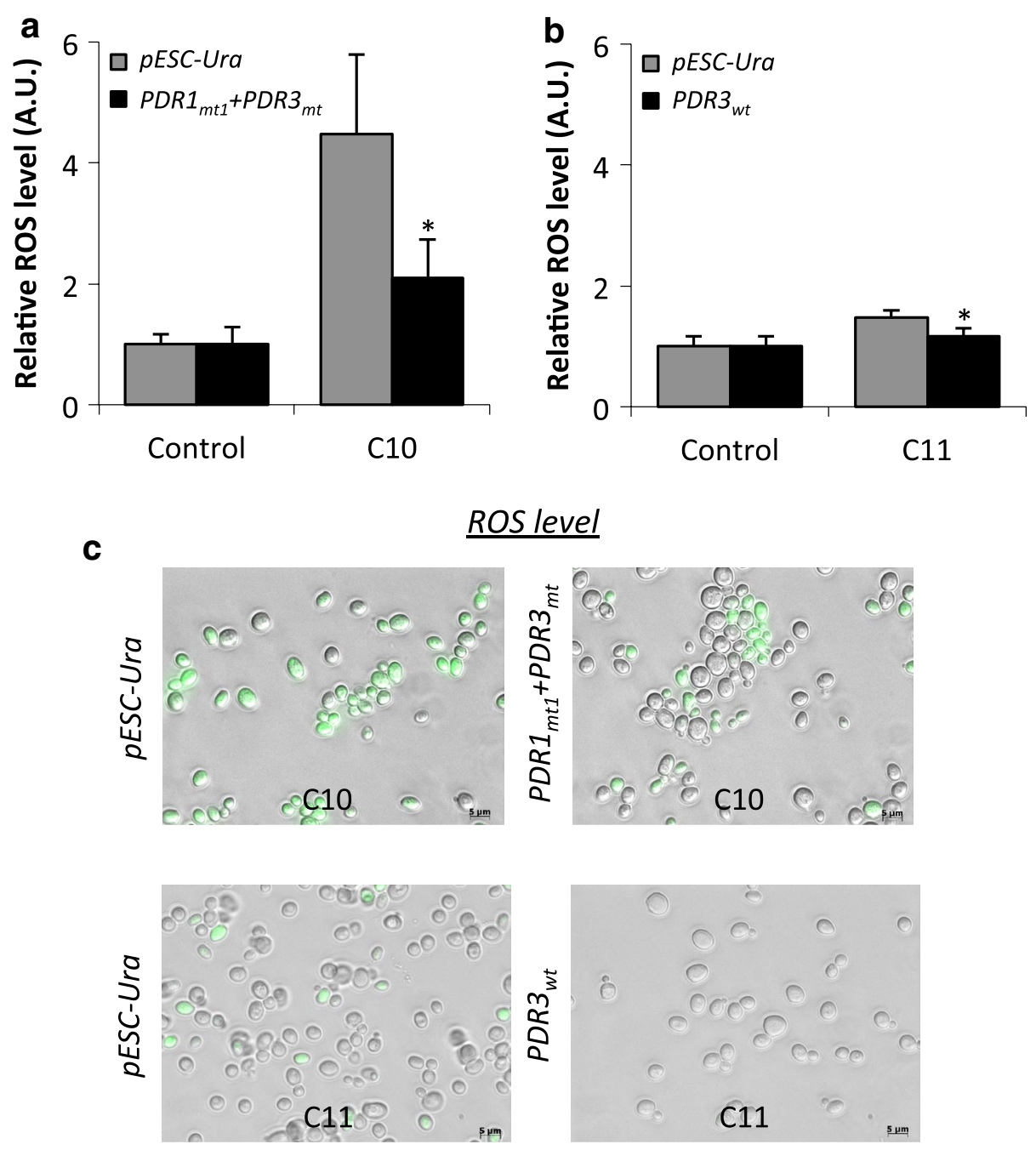

Fig. 5 Quantification of ROS levels in BYL13 expressing Pdr transcription factors. a and $\mathbf{b}$ ROS levels upon exposure to C10 and C11 alkanes. The relative ROS levels of BYL13 without alkane were set to 1. c Comparison of fluorescence images, where stronger green fluorescence indicates higher ROS levels. AU arbitrary unit. Asterisk significant difference (one-tailed Student $t$ test, $\mathrm{p}<0.05$ ); error bars SD from three biological replicates

of $\mathrm{C} 10$ alkane. However, Fig. $6 \mathrm{~b}$ shows that, in the presence of C11 alkane, both BYL13 with Pdr3 $3_{\mathrm{wt}}$ and the control cells had comparable RFU ratios, suggesting intact cell membrane in the presence of C11 alkane.

The low RFU ratio suggests that BYL13 expressing $\operatorname{Pdr} 1_{\mathrm{mt}}+\operatorname{Pdr} 3_{\mathrm{mt}}$ had less membrane damage than the control cells in the presence of $\mathrm{C} 10$ alkane, and the comparable ratios suggest no membrane damage to both BYL13 expressing Pdr $3_{\mathrm{wt}}$ and the control cells in the presence of C11 alkane, in line with the fluorescence microscopy images in Fig. 6c.

In this study, we improved yeast's alkane tolerance by expressing wild-type or site-mutated Pdr transcription factors in $S$. cerevisiae $p d r 1 \Delta p d r 3 \Delta$, and provided the evidence that, in the most tolerant strains expressing
Pdr transcription factors, (i) a series of genes (e.g., ABC efflux pump genes, CTT1, and ICT1) were up-regulated by $\mathrm{C} 10$ and $\mathrm{C} 11$ alkanes; (ii) intracellular alkane levels were reduced over $67 \%$ due to alkane efflux and/or low import; and (iii) ROS levels were reduced over $21 \%$; and (iv) cell membrane damage was also reduced. However, expression of any Pdr transcription factors did not improve tolerance to C8 or C9 alkanes at toxic levels. The susceptibility of yeast to alkanes is associated with multiple factors such as alkane carbon-chain length, alkane concentration, and strain background. Additional file 1: Figure S2 shows that, more C8 and C9 alkanes were required to inhibit BYL13 with pESC-Ura than C10 alkane, although $\mathrm{C} 8$ and $\mathrm{C} 9$ alkanes are more toxic than $\mathrm{C} 10$ and $\mathrm{C} 11$ alkanes. This could be because $\mathrm{C} 8$ and 

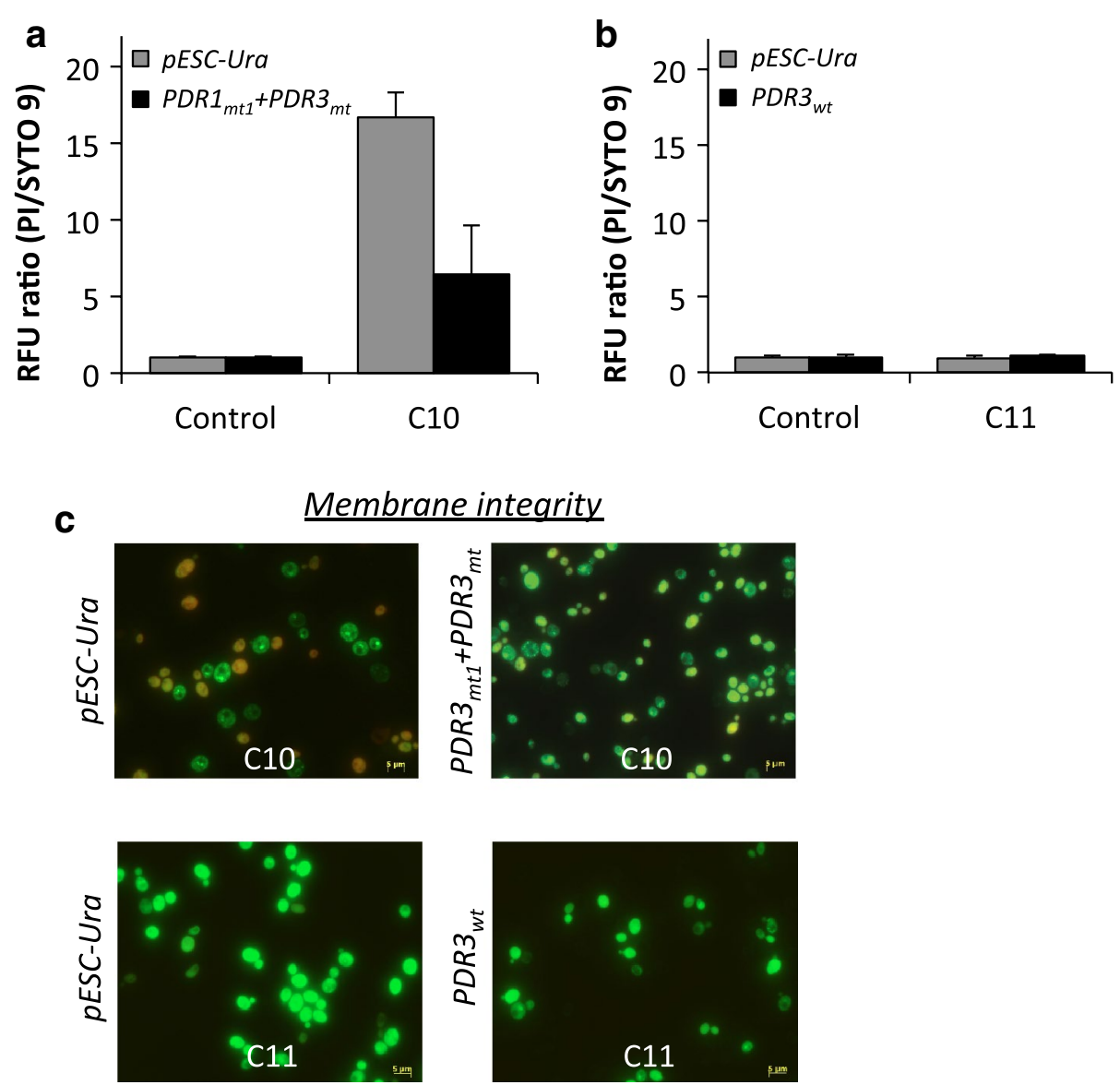

Fig. 6 Assays of membrane integrity in BYL13 expressing Pdr transcription factors. a and $\mathbf{b}$ RFU ratios of PI and SYTO 9 upon exposure to C10 and C11 alkanes. The relative RFU ratios of BYL13 without alkane were set to 1. c Representative fluorescent images. Error bars SD from three biological replicates

C9 alkanes are more volatile than longer-chain alkanes. Furthermore, although Pdr1p and Pdr3p improved tolerance to C10 and C11 alkanes in BYL13, we found that Pdr1 R821S ( $\mathrm{Pdr} 1_{\mathrm{mt} 2}$ ) could not improve tolerance to C9 alkane, which is inconsistent with a previous study [21]. This discrepancy is likely due to difference of strain background between BYL13 and KK-211 used in the previous study. To demonstrate that strain background can affect cell tolerance towards alkanes, we expressed either $\operatorname{Pdr} 1_{\mathrm{mt} 1}+\mathrm{Pdr} 3_{\mathrm{mt}}$ or $\mathrm{Pdr} 3_{\mathrm{wt}}$ in BY4741, a parental strain of BYL13, and evaluated the cell growth in the presence of alkanes. Figure 2 and Additional file 1: Figure S5 show that BY4741 expressing Pdr transcription factors grew better than BYL13 expressing the same Pdr transcription factors upon exposure to $\mathrm{C} 10$ and $\mathrm{C} 11$ alkanes.

According to Mamnun and coworkers [22], Pdr1p and Pdr3p form homo- and hetero-dimers to mediate pleiotropic drug resistance in S. cerevisiae, and these homoand hetero-dimers could show diverse transcriptional activity to their target genes involved in tolerance to alkanes. In line with the diversity of Pdr dimers and their transcriptional activity, Fig. 2 and Additional file 1: Figure S3 indicate discrepant tolerance conferred by the individual and co-expressed Pdr transcription factors.

Future efforts could be made to identify DNA-binding efficacy of Pdr1p-and Pdr3p-dimers as well as influences of various Pdr dimers on the transcriptome in response to alkanes, and to discriminate significance of each mechanism (alkanes efflux, membrane modifications, ROS reduction, and alleviation of membrane damage) in the Pdr transcription factors involving tolerance improvement towards alkane biofuels. In addition, a tool of global transcription machinery engineering (gTME) [19] can be applied to construct Pdr transcription factor libraries and obtain phenotypes of resistance against a wide spectrum of biochemical molecules.

\section{Conclusions}

The site mutants Pdr1 F815S + Pdr3 Y276H $\left(\operatorname{Pdr} 1_{\mathrm{mt1}}+\operatorname{Pdr} 3_{\mathrm{mt}}\right)$ and the wild-type Pdr3p $\left(\operatorname{Pdr} 3_{\mathrm{wt}}\right)$ 


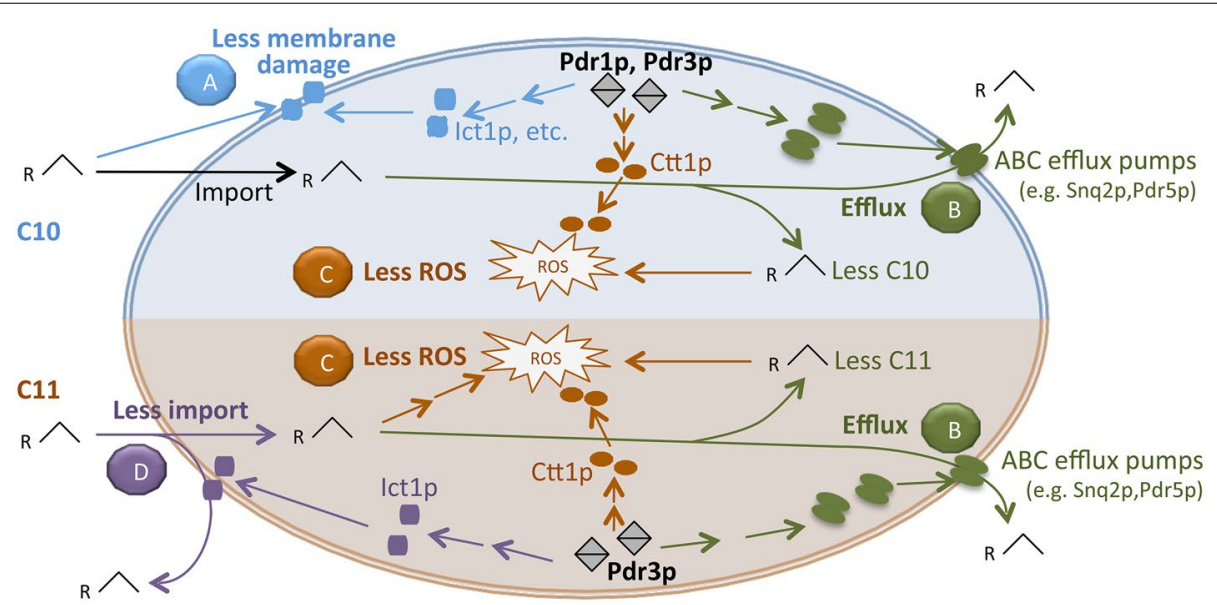

Fig. 7 A schematic of proposed mechanisms on tolerance improvement towards C10 (shaded in light blue) and C11 (shaded in light orange) alkanes conferred by Pdr1p and Pdr3p. Pdr1 p and Pdr3p improve tolerance to C10 alkane likely through reducing membrane damage ( $A$, blue), alkane efflux $(B$, green), and reducing ROS production (C, orange); the tolerance to $C 11$ alkane is improved likely through reducing $C 11$ import due to potential membrane modifications ( $D$, purple), as well as $(B)$ and $(C)$

improved the tolerance of BYL13 and BY4741 to C10 and C11 alkanes, respectively. We found that the mechanisms underlying Pdr1p- and Pdr3p-mediated tolerance are multilayered. As depicted in Fig. 7, we hypothesize that Pdr1p and Pdr3p regulate genes involved in alkane efflux (e.g., SNQ2, and PDR5), stress responses (e.g., CTT1) and membrane modifications (e.g., ICT1) in the presence of $\mathrm{C} 10$ and $\mathrm{C} 11$ alkanes. The tolerance to alkanes was improved through (i) reduced intracellular alkanes contributed by alkane efflux (C10 and $\mathrm{C} 11)$ and lower alkane import (C11 alkane), (ii) decreased ROS production probably contributed by lower alkane accumulation (C10 and C11 alkanes) and Ctt1p-mediated ROS decomposition (C10 and $\mathrm{C} 11$ alkanes), and (iii) alleviated membrane damage contributed to by membrane modifications (C10 alkane). These findings provide valuable insights into engineering alkane-tolerant yeast for improved alkane productivity.

\section{Methods}

\section{Strains, plasmids and growth conditions}

Strains and plasmids used in this study are listed in Table 1 . Yeast cells were grown at $30{ }^{\circ} \mathrm{C}$ in YPD $(10 \mathrm{~g} / \mathrm{l}$ Yeast Extract, $20 \mathrm{~g} / \mathrm{l}$ Peptone, and $20 \mathrm{~g} / \mathrm{l}$ Dextrose), minimal medium (6.7 g/l Yeast Nitrogen Base, $20 \mathrm{~g} / \mathrm{l}$ Dextrose, and $1.92 \mathrm{~g} / \mathrm{l}$ Yeast synthetic dropout medium supplements without uracil), or induction medium (6.7 g/l Yeast Nitrogen Base, 10 g/l D-raffinose, 1.92 g/l Yeast synthetic dropout medium supplements without uracil, and appropriate amount of D-(+)-galactose). E. coli was grown at $37^{\circ} \mathrm{C}$ in Luria-Bertani (LB) broth. Antibiotics $(200 \mu \mathrm{g} / \mathrm{ml}$
G418, or $100 \mu \mathrm{g} / \mathrm{ml}$ Ampicillin) and appropriate amount of n-alkanes were added if necessary.

\section{Disruption of $P D R 1$ and PDR3}

Gene disruption was carried out as previously described [31] using primers listed in Additional file 2: Table S3. First, gene disruption cassettes of loxP-Kan-loxP were amplified from pUG6 and transformed into $S$. cerevisiae BY4741. Colonies were screened on YPD agar plates containing $200 \mu \mathrm{g} / \mathrm{ml} \mathrm{G418.} \mathrm{Subsequently,} \mathrm{pSH47} \mathrm{was} \mathrm{trans-}$ formed into a single mutant BYL1K, and transformants were screened on minimal medium $\left(\mathrm{Ura}^{-}\right)$plates, followed by Cre/loxP-mediated marker removal in galactose-induction medium. Selection for loss of the marker was performed in YPD containing $1 \mathrm{mg} / \mathrm{ml} 5$-fluoro-orotic acid (Thermo Scientific). Finally, marker-free $p d r 1 \Delta$ (BYL1) was used as a parental strain for disruption of PDR3.

\section{Gene cloning and expression}

With S. cerevisiae BY4741 genomic DNA as a template, wild-type PDR1 and PDR3 were amplified using iProof High-Fidelity DNA Polymerase (Biorad) and genespecific primers (Additional file 2: Table S3). A-tailing of PCR products was performed with Taq polymerase (New England Biolabs). The PCR products were purified, cloned into pGEM-T (Promega) and confirmed by sequencing. Subsequently, correct recombinant plasmids were used as templates for site mutagenesis using Quick Change Site-Directed Mutagenesis Kit (Stratagene) according to a provided manual. Further, site mutations 
Table 1 Strains, plasmids and PDR alleles used in this study

\begin{tabular}{|c|c|c|}
\hline Strains, plasmids, and genes & Description & Sources \\
\hline \multicolumn{3}{|l|}{ S. cerevisiae strains } \\
\hline BY4741 & 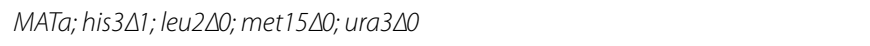 & ATCC201388 \\
\hline BYL1K & pdr1::kanMX derived from BY4741 & This study \\
\hline BYL13K & pdr3::kanMX derived from BYL1K & This study \\
\hline BYL13 & $p d r 1 \Delta p d r 3 \Delta$, KanMX-free BYL13K & This study \\
\hline \multicolumn{3}{|l|}{ Plasmids } \\
\hline pESC-Ura & Expression vector, $\mathrm{Ura}^{+}$ & Agilent \\
\hline pRS42K & Expression vector, $\mathrm{Kan}^{+}$ & Euroscarf \\
\hline $\mathrm{pSH} 47$ & A plasmid for marker rescue, $\mathrm{Ura}^{+}$ & Euroscarf \\
\hline pUG6 & A plasmid with a KanMX gene cassette, $\mathrm{Amp}^{+}$ & Euroscarf \\
\hline \multicolumn{3}{|l|}{$P D R 1$ and $P D R 3$ alleles } \\
\hline$P D R 1_{w t}$ & Wild-type PDR1 & This study \\
\hline$P D R 1_{m t 1}$ & A site mutant of $P D R 1$ with amino acid substitution of F815S & This study, [24] \\
\hline$P D R 1_{m+2}$ & A site mutant of PDR1 with amino acid substitution of R821S & This study, [21] \\
\hline$P D R 1_{m t 1+2}$ & A site mutant of PDR1 with amino acid substitution of both F815S and R821S & This study \\
\hline$P D R 3_{w t}$ & Wild-type PDR3 & This study \\
\hline$P D R 3_{m t}$ & A site mutant of PDR3 with amino acid substitution of $Y 276 \mathrm{H}$ & This study, [23] \\
\hline
\end{tabular}

were confirmed by sequencing. Thereafter, wild-type and site mutants of PDR1 and PDR3 were amplified and cotransformed with linearized pESC-Ura fragments into BYL13 by an in vivo DNA assembly method [32]. Finally, transformants were screened on minimal medium agar plates $\left(\mathrm{Ura}^{-}\right)$and characterized by PCR and sequencing. Here, PDR 1 and its site mutants were cloned into multiple cloning site 2 (MCS2) under control of a galactose-inducible promoter $P_{G A L 1}$, while PDR3 and its site mutant were cloned into MCS1 under control of another galactose-inducible promoter $P_{G A L 10}$. These PDR genes in MCS1 and MCS2 were individually expressed or coexpressed under induction by galactose (Fig. 1).

\section{Western blotting}

Pdr proteins were induced in the induction medium containing $0.5 \mathrm{~g} / \mathrm{l}$ galactose for $24 \mathrm{~h}$. Fifty milliliters of cells were collected and resuspended in ice-cooled lysis buffer (50 mM Tris- $\mathrm{HCl}$ pH7.9, 0.6 M sorbitol) added with protease inhibitor and $0.3 \mathrm{~g}$ acid-washed glass beads (diameter 425-600 $\mu \mathrm{m}$ ). Cells were disrupted using FastPrep- 24 at $6 \mathrm{~m} / \mathrm{s}$ for $30 \mathrm{~s} \times 10$ cycles (MP Biomedicals, USA). Samples were chilled on ice for 5 min during each interval. Cell lysate was centrifuged at $4{ }^{\circ} \mathrm{C}$ to separate soluble and insoluble proteins. The insoluble part was dissolved with $8 \mathrm{M}$ urea. The obtained protein samples were separated by SDS-PAGE, transferred onto nitrocellulose membrane, and hybridized with anti-Myc (for Pdr1p) and anti-Flag (for Pdr3p) antibodies (Abcam). Protein bands with positive signal were detected using SuperSignal West Pico Chemiluminescent Substrate (Thermo Scientific).

\section{Tolerance tests}

First, we determined conditions of protein induction. In induction media with $20 \mathrm{~g} / \mathrm{l}$ galactose, we measured growth curves of BYL13 expressing Pdr transcription factors, and a strain with minimal growth was selected to optimize galactose concentrations. Subsequently, the selected strain was grown in induction media with galactose $(0.5 \mathrm{~g} / \mathrm{l}, 5 \mathrm{~g} / \mathrm{l}$, and $20 \mathrm{~g} / \mathrm{l})$. Every $12 \mathrm{~h}, 100 \mu \mathrm{l}$ cell culture was sampled to measure cell densities $\left(\mathrm{OD}_{600}\right)$ by a BioTek microplate reader, and galactose concentration which gave low growth inhibition was chosen for tolerance tests. Second, we determined conditions of alkanes exposure as follows. BYL13 with pESC-Ura was grown in induction media with galactose at the previously determined concentration, and exposed to alkanes (v/v, C8: 2, 5, 10 \%, C9: 1, 2, 5 \%, C10: 0.5, 1, 5, and C11: 5, 10, $20 \%)$. Based on growth curves, minimum concentrations of alkanes that inhibited BYL13 with pESC-Ura were selected for tolerance tests. Third, to test tolerance of cells with Pdr transcription factors against alkanes, we grew the cells in induction media under the determined conditions for protein induction and alkanes exposure. Strains showing the highest cell densities were selected for further analyses.

\section{Total RNAs extraction and quantitative PCR analyses}

BYL13 expressing Pdr1 F815S/Pdr3 Y276H $\left(\operatorname{Pdr} 1_{\mathrm{mt} 1}+\mathrm{Pdr} 3_{\mathrm{mt}}\right)$ and wild-type Pdr3p $\left(\mathrm{Pdr} 3_{\mathrm{wt}}\right)$ were exposed to $1 \% \mathrm{C} 10$ and $5 \% \mathrm{C} 11$, respectively, for $6 \mathrm{~h}$. Thereafter, the treated cells were collected, and cell wall was disrupted by Lyticase (Sigma). Total RNAs were 
extracted using an RNeasy Mini Kit (Qiagen) according to a provided manual. Single-stranded cDNAs were synthesized from $1 \mu \mathrm{g}$ total RNA using a RevertAid First Strand cDNA Synthesis Kit (Thermal Scientific). With an equal amount of cDNAs, qPCR was performed using gene-specific primers (Additional file 2: Table S3) and Biorad SsoFast EvaGreen Supermix, and fluorescence signals were detected and analyzed by a Biorad iQ5 optical system. Expression stability of five reference gene candidates (ACT1, ALG9, TAF10, TFC1, and UBC6) was evaluated subjective to $M$ value $[26,27]$. Finally, gene expression data were normalized to a reference gene UBC6 and controls. Here, samples from BYL13 with pESC-Ura and alkanes exposure were used as controls.

\section{Alkane extraction and quantification}

Alkanes were extracted by a chloroform-methanol extraction method [33] with modifications. First, BYL13 expressing $\mathrm{Pdr} 1_{\mathrm{mt} 1}+\mathrm{Pdr}_{\mathrm{mt}}$ and BYL13 expressing Pdr3 $3_{\mathrm{wt}}$ were exposed to $1 \% \mathrm{C} 10$ and $5 \% \mathrm{C} 11$, respectively. Thereafter, the alkane-treated cells were collected, washed with $50 \mathrm{mM}$ Tris- $\mathrm{Cl}(\mathrm{pH}$ 7.5), and resuspended in $0.5 \mathrm{ml}$ chloroform-methanol mixture (v/v: 2/1). With $0.01 \% \mathrm{n}$-dodecane $(\mathrm{C} 12)$ and $0.3 \mathrm{~g}$ glass beads per sample, cells were lysed by FastPrep-24, and lysate was separated by centrifugation at a top speed for $10 \mathrm{~min}$ at $4{ }^{\circ} \mathrm{C}$. Supernatant was collected, and alkanes were extracted by adding appropriate amount of chloroform and $50 \mathrm{mM}$ Tris-Cl (pH7.5). Finally, chloroform phases containing alkanes were injected into a GC 7890A system and analyzed under conditions as follows: with a HP-5 column (Agilent Technologies), oven temperature started at $80{ }^{\circ} \mathrm{C}$, held for $1 \mathrm{~min}$, ramped at $20^{\circ} \mathrm{C} / \mathrm{min}$ until $180{ }^{\circ} \mathrm{C}$, and held for $2 \mathrm{~min}$; FID detector temperature remained at $275^{\circ} \mathrm{C}$. A mixture of n-alkanes (10 ppm each) was used as a standard. Finally, areas of GC peaks were normalized to an internal standard and corresponding protein amount.

To investigate involvement of alkane efflux in reducing intracellular alkane amount, BYL13 expressing $\mathrm{Pdr} 1_{\mathrm{mt} 1}+\mathrm{Pdr} 3_{\mathrm{mt}}$ and BYL13 expressing Pdr $3_{\mathrm{wt}}$ were grown until $\log$ phases and exposed to $1 \% \mathrm{C} 10$ and $5 \%$ $\mathrm{C} 11$, respectively, for $1 \mathrm{~h}$. Cells were washed and divided into two equal aliquots. The first aliquot was treated with $1 \mathrm{mM} \mathrm{NaN}_{3}$ for $80 \mathrm{~min}$, to deactivate activities of $\mathrm{ABC}$ transporters [34]. Here, $\mathrm{NaN}_{3}$ functions as a metabolic inhibitor that interferes with $\mathrm{ABC}$ transporters by decreasing the amount of ATP generated by mitochondria. The second aliquot was incubated without $\mathrm{NaN}_{3}$ for $80 \mathrm{~min}$. Finally, alkanes were extracted and quantified.

To investigate involvement of alkanes import in reducing intracellular alkane amount, BYL13 expressing Pdr transcription factors (i.e., Pdr $1_{\mathrm{mt} 1}+\mathrm{Pdr} 3_{\mathrm{mt}}$, and $\mathrm{Pdr} 3_{\mathrm{wt}}$ ) and BYL13 with pESC-Ura (control), respectively, were grown in an alkane-free induction medium until log phases and treated with $1 \mathrm{mM} \mathrm{NaN}_{3}$ for 20 min to deactivate activities of $A B C$ transporters [34]. An equal amount of the $\mathrm{NaN}_{3}$-treated cells were subsequently exposed to $1 \% \mathrm{C} 10$ and $5 \% \mathrm{C} 11$, respectively, for $1 \mathrm{~h}$. After exposure, alkanes were extracted and quantified.

\section{ROS quantification and membrane integrity analyses} BYL13 expressing Pdr $1_{\mathrm{mt1}}+\mathrm{Pdr}_{\mathrm{mt}}$ and BYL13 expressing $\mathrm{Pdr} 3_{\mathrm{wt}}$ at $\log$ phases were exposed to $1 \% \mathrm{C} 10$ and $5 \%$ $\mathrm{C} 11$, respectively, for $6 \mathrm{~h}$. Thereafter, the alkane-treated cells were collected and stained by CellROX ${ }^{\circledR}$ Green Reagent (Life Technologies) for ROS analyses, and by SYTO 9 and propidium iodide (PI) (Life Technologies) for membrane integrity analyses. Further, fluorescence signals from CellROX ${ }^{\circledR}$ Green Reagent were measured by a TECAN Infinite 200 microplate reader at wavelength of 485 nm (Excitation, Ex)/535 nm (Emission, Em), while fluorescence signals from PI and SYTO 9 were measured at wavelength of $535 \mathrm{~nm}(\mathrm{Ex}) / 590 \mathrm{~nm}(\mathrm{Em})$ and $485 \mathrm{~nm}(\mathrm{Ex}) / 535 \mathrm{~nm}(\mathrm{Em})$, respectively. The acquired fluorescence signals were normalized to cell densities $\left(\mathrm{OD}_{600}\right)$ if necessary. In addition, the stained cells were observed at wavelength of $535 \mathrm{~nm}(\mathrm{Ex}) / 590 \mathrm{~nm}(\mathrm{Em})$ and $470 \mathrm{~nm}(\mathrm{Ex}) / 525 \mathrm{~nm}(\mathrm{Em})$ under a Zeiss Axio Scope.A1 microscope.

\section{Additional files}

Additional file 1. Additional figures. Figure S1. Growth inhibition by expression of the site-mutants of Pdr transcription factors, and determination of suitable galactose concentrations. Figure S2. Determination of alkane exposure concentrations. Figure S3. Growth of BYL13 expressing Pdr transcription factors in the presence of alkanes. Figure S4. Western blotting of Pdr proteins in BYL13. Figure S5. Tolerance of BY4741 expressing Pdr transcription factors against C10 and C11 alkanes.

Additional file 2. Additional tables.Table S1. M values of reference gene candidates derived from qPCR data. Table S2. Fold changes of PDR genes expressed in BYL13 stains as compared to BY4741. Table S3. Primers used in this study.

\section{Abbreviations}

PDR: pleiotropic drug resistance; Wt: wild type; Mt: mutant; C8: n-octane; C9: n-undecane; C10: n-decane; C11: n-undecane; C12: n-dodecane; ROS: reactive oxygen species; ABC: ATP-binding cassette; MCS: multiple cloning sites; PI: propidium iodide; qPCR: quantitative PCR; RFU: relative fluorescence unit

\section{Authors' contributions}

$\mathrm{HL}$ and MWC conceived the project and designed the experiments. HL carried out gene disruption, ROS and membrane integrity analyses, and participated in alkane detection and tolerance tests. NKPJ carried out Western blotting and participated in tolerance tests. WST carried out gene cloning and participated in alkane detection. RL carried out RNA extraction and GPCR analyses. HL, SSJL and MWC wrote the manuscript. MWC supervised the project. All authors read and approved the final manuscript.

\section{Author details}

${ }^{1}$ Department of Biochemistry, Yong Loo Lin School of Medicine, National University of Singapore, 8 Medical Drive, 117597 Singapore, Singapore. 
${ }^{2}$ NUS Synthetic Biology for Clinical and Technological Innovation (SynCTI), Life Sciences Institute, National University of Singapore, 28 Medical Drive, 117456 Singapore, Singapore. ${ }^{3}$ Singapore Institute of Technology, 10 Dover Drive, 138683 Singapore, Singapore.

\section{Acknowledgements}

This study was financially supported by the Competitive Research Program of the National Research Foundation of Singapore (NRF-CRP5-2009-03), and the Defense Threat Reduction Agency (DTRA, HDTRA1-13-1-0037).

\section{Competing interests}

The authors declare that they have no competing interests.

Received: 31 August 2015 Accepted: 4 December 2015

Published online: 30 December 2015

\section{References}

1. Peralta-Yahya PP, Zhang F, del Cardayre SB, Keasling JD. Microbial engineering for the production of advanced biofuels. Nature. 2012;488:320-8.

2. Blazeck J, Liu L, Knight R, Alper HS. Heterologous production of pentane in the oleaginous yeast Yarrowia lipolytica. J Biotechnol. 2013;165:184-94.

3. Yang X, Dai X, Guo H, Geng S, Wang G. Petrodiesel-like straight chain alkane and fatty alcohol production by the microalga Chlorella sorokiniana. Bioresour Technol. 2013;136:126-30.

4. Andre C, Kim SW, Yu XH, Shanklin J. Fusing catalase to an alkaneproducing enzyme maintains enzymatic activity by converting the inhibitory byproduct $\mathrm{H}_{2} \mathrm{O}_{2}$ to the cosubstrate O2. Proc Natl Acad Sci USA. 2013;110:3191-6.

5. Schirmer A, Rude MA, Li X, Popova E, del Cardayre SB. Microbial biosynthesis of alkanes. Science. 2010;329:559-62.

6. Akhtar MK, Turner NJ, Jones PR. Carboxylic acid reductase is a versatile enzyme for the conversion of fatty acids into fuels and chemical commodities. Proc Natl Acad Sci USA. 2013;110:87-92.

7. Bernard A, Domergue F, Pascal S, Jetter R, Renne C, Faure JD, Haslam RP, Napier JA, Lessire R, Joubes J. Reconstitution of plant alkane biosynthesis in yeast demonstrates that Arabidopsis ECERIFERUM1 and ECERIFERUM3 are core components of a very-long-chain alkane synthesis complex. Plant Cell. 2012;24:3106-18.

8. Choi YJ, Lee SY. Microbial production of short-chain alkanes. Nature. 2013;502:571-4.

9. Rasouliha $\mathrm{BH}$, Ling $\mathrm{H}, \mathrm{Ho} \mathrm{CL}$, Chang MW. A predicted immunity protein confers resistance to pyocin $\mathrm{S} 5$ in a sensitive strain of Pseudomonas aeruginosa. ChemBioChem. 2013;14:2444-6.

10. Chen $B$, Ling $H$, Chang MW. Transporter engineering for improved tolerance against alkane biofuels in Saccharomyces cerevisiae. Biotechnol Biofuels. 2013;6:21.

11. Dunlop MJ, Dossani ZY, Szmidt HL, Chu HC, Lee TS, Keasling JD, Hadi MZ, Mukhopadhyay A. Engineering microbial biofuel tolerance and export using efflux pumps. Mol Syst Biol. 2011;7:487.

12. Lo TM, Teo WS, Ling H, Chen B, Kang A, Chang MW. Microbial engineering strategies to improve cell viability for biochemical production. Biotechnol Adv. 2013;31:903-14.

13. Lin Y, Chomvong K, Acosta-Sampson L, Estrela R, Galazka JM, Kim SR, Jin Y-S, Cate JH. Leveraging transcription factors to speed cellobiose fermentation by Saccharomyces cerevisiae. Biotechnol Biofuels. 2014;7:126.

14. Ling H, Teo W, Chen B, Leong SS, Chang MW. Microbial tolerance engineering toward biochemical production: from lignocellulose to products. Curr Opin Biotechnol. 2014;29C:99-106.

15. Kang A, Tan MH, Ling H, Chang MW. Systems-level characterization and engineering of oxidative stress tolerance in Escherichia coli under anaerobic conditions. Mol BioSyst. 2013;9:285-95.
16. Tamayo-Ramos J, Orejas M. Enhanced glycosyl hydrolase production in Aspergillus nidulans using transcription factor engineering approaches. Biotechnol Biofuels. 2014;7:103.

17. Alper H, Moxley J, Nevoigt E, Fink GR, Stephanopoulos G. Engineering yeast transcription machinery for improved ethanol tolerance and production. Science. 2006;314:1565-8.

18. Ghosh AK, Ramakrishnan G, Rajasekharan R. YLR099C (ICT1) encodes a soluble Acyl-CoA-dependent lysophosphatidic acid acyltransferase responsible for enhanced phospholipid synthesis on organic solvent stress in Saccharomyces cerevisiae. J Biol Chem. 2008;283:9768-75.

19. Alper H, Stephanopoulos G. Global transcription machinery engineering: a new approach for improving cellular phenotype. Metab Eng. 2007:9:258-67.

20. Kang A, Chang MW. Identification and reconstitution of genetic regulatory networks for improved microbial tolerance to isooctane. Mol BioSyst. 2012:8:1350-8.

21. Matsui K, Teranishi S, Kamon S, Kuroda K, Ueda M. Discovery of a modified transcription factor endowing yeasts with organic-solvent tolerance and reconstruction of an organic-solvent-tolerant Saccharomyces cerevisiae strain. Appl Environ Microbiol. 2008;74:4222-5.

22. Mamnun YM, Pandjaitan R, Mahe Y, Delahodde A, Kuchler K. The yeast zinc finger regulators $\mathrm{Pdr} 1 \mathrm{p}$ and $\mathrm{Pdr} 3 \mathrm{p}$ control pleiotropic drug resistance (PDR) as homo- and heterodimers in vivo. Mol Microbiol. 2002;46:1429-40.

23. Nourani A, Papajova D, Delahodde A, Jacq C, Subik J. Clustered amino acid substitutions in the yeast transcription regulator Pdr3p increase pleiotropic drug resistance and identify a new central regulatory domain. Mol Gen Genet. 1997;256:397-405.

24. Carvajal E, van den Hazel HB, Cybularz-Kolaczkowska A, Balzi E, Goffeau A. Molecular and phenotypic characterization of yeast PDR1 mutants that show hyperactive transcription of various $A B C$ multidrug transporter genes. Mol Gen Genet. 1997;256:406-15.

25. Mizoguchi $H$, Yamauchi T, Watanabe $M$, Yamanaka $H$, Nishimura $A$ Hanamoto H. Different missense mutations in PDR1 and PDR3 genes from clotrimazole-resistant sake yeast are responsible for pleiotropic drug resistance and improved fermentative activity. J Biosci Bioeng. 2002;93:221-7.

26. Teste MA, Duquenne M, Francois JM, Parrou JL. Validation of reference genes for quantitative expression analysis by real-time RT-PCR in Saccharomyces cerevisiae. BMC Mol Biol. 2009;10:99.

27. Vandesompele J, De Preter K, Pattyn F, Poppe B, Van Roy N, Paepe A, Speleman F. Accurate normalization of real-time quantitative RT-PCR data by geometric averaging of multiple internal control genes. Genome Biol. 2002;3:RESEARCH0034.

28. Jungwirth $\mathrm{H}$, Kuchler K. Yeast $A B C$ transporters - a tale of sex, stress, drugs and aging. FEBS Lett. 2006;580:1131-8.

29. Kim HS, Kim NR, Kim W, Choi W. Insertion of transposon in the vicinity of SSK2 confers enhanced tolerance to furfural in Saccharomyces cerevisiae. Appl Microbiol Biotechnol. 2012;95:531-40.

30. Sikkema J, de Bont JA, Poolman B. Mechanisms of membrane toxicity of hydrocarbons. Microbiol Rev. 1995;59:201-22.

31. Gueldener U, Heinisch J, Koehler GJ, Voss D, Hegemann JH. A second set of loxP marker cassettes for Cre-mediated multiple gene knockouts in budding yeast. Nucleic Acids Res. 2002;30:e23.

32. Shao Z, Zhao H, Zhao H. DNA assembler, an in vivo genetic method for rapid construction of biochemical pathways. Nucleic Acids Res. 2009;37:e16.

33. Maeng JH, Sakai Y, Tani Y, Kato N. Isolation and characterization of a novel oxygenase that catalyzes the first step of n-alkane oxidation in Acinetobacter sp. strain M-1. J Bacteriol. 1996;178:3695-700.

34. Wilcox LJ, Balderes DA, Wharton B, Tinkelenberg AH, Rao G, Sturley SL. Transcriptional profiling identifies two members of the ATP-binding cassette transporter superfamily required for sterol uptake in yeast. J Biol Chem. 2002;277:32466-72. 